\title{
EL ARBITRAJE ESTADÍSTICO Y SU INFLUENCIA EN LA CALIDAD DE LAS PUBLICACIONES CIENTÍFICAS
}

\author{
Verena Torres Cárdenas ${ }^{1} \quad$ Rafael Herrera García $^{1} \quad$ Lucía Sarduy García $^{1}$ \\ Recibido el 28 de septiembre de 2004, aceptado el 21 de abril de 2005
}

\begin{abstract}
RESUMEN
En el proceso de investigación, la aplicación de métodos estadísticos adecuados en la etapa de presentación y publicación de los resultados desempeña un importante papel, por cuanto garantiza la precisión y exactitud de las estimaciones y conclusiones. Este trabajo, continuación del presentado en el IV Encuentro de Editores de Revistas Científicas (La Habana, 2002), tiene como objetivo continuar señalando las anomalías encontradas en la metodología experimental descritas en muchas de las revistas científicas de América Latina y el Caribe editadas entre los años 2000 y 2004. Se resumen los aspectos de mayor influencia negativa relacionados con los diseños experimentales clásicos. Las deficiencias que con mayor frecuencia se observan son, entre otras: no se especifica el paquete estadístico utilizado para el procesamiento de la información (62\%); no aparece el error estándar en las tablas (51\%); incorrecta presentación de los elementos estadísticos en las tablas (33\%), e inapropiada descripción del diseño experimental (30\%). En la actualidad es un desafío, para todas la ediciones científicas, el satisfacer las expectativas de los usuarios relacionadas con las herramientas estadísticas que se aplican, y resulta imprescindible realizar un esfuerzo y emplear todas las posibilidades para lograr indicadores de calidad en las publicaciones, sobre todo en las revistas científicas de la región.
\end{abstract}

Palabras clave: Calidad, arbitraje, publicaciones científicas.

\section{ABSTRACT}

The application of adequate statistical methods at the moment of presenting and publishing the scientific results plays an important role in the research process because they guarantee the accuracy and precision of the estimations and the conclusions. This paper is a continuity of that presented in the IV Meeting of Editors of Scientific Journals held in Havana in 2002 and its purpose is to continue revealing the difficulties in the experimental methodology described in many scientific journals of Latin America and the Caribbean that were edited between 2000 and 2004. This paper summarizes the aspects related to the classical experimental designs of greatest negative influence. The deficiencies that are observed more frequently are, among others, the lack of specification of the statistical package used for data processing (62\%), not mentioning the standard error in the tables (51\%), the incorrect presentation of the statistical elements in the tables $(33 \%)$ and the inappropriate description of the experimental design $(30 \%)$. At present, the fulfilment of the reader's expectancies related to the statistical tools applied is a challenge for all the scientific editions and it is indispensable to make an effort and use all the possibilities at hand to produce indicators of quality in the publications, mainly in the scientific journals of the region.

Keywords: Quality, review, scientific publications.

\section{INTRODUCCIÓN}

La comunidad científica de lengua española realiza esfuerzos para lograr un sistema de evaluación del impacto y la visibilidad de las publicaciones científicas. Para ello es necesario aumentar la calidad de los artículos que se publican.
Las publicaciones científicas latinoamericanas siguen constituyendo, aún hoy, un universo poco conocido, en especial debido a factores tales como: la escasa presencia de los materiales en servicios internacionales, regionales e incluso nacionales de información; normalmente es limitada su distribución en bibliotecas

\footnotetext{
1 Instituto de Ciencia Animal, Carretera Central km 47.4, San José de las Lajas, La Habana, Cuba. vtorres@ica.co.cu, rherrera@ica.co.cu, lsarduy@ica.co.cu
} 
y centros de documentación, tanto dentro como fuera de la región; poco reconocimiento por parte de la comunidad científica internacional, a pesar de la relevancia que puedan tener [1].

Los Encuentros de Editores de Revistas Científicas, que se celebran cada dos años en Cuba, han permitido analizar y conocer el trabajo que se realiza en el área para subsanar esta situación. Han sido expuestos resultados de México, Argentina, Honduras, Colombia, Costa Rica, Chile, Brasil, Venezuela y Cuba y entre los esfuerzos más importantes están el proyecto Latindex y el modelo Scielo [6].

Uno de los aspectos que influyen en la calidad de los artículos científicos es el relacionado con la metodología estadística utilizada y la presentación de los resultados. Su importancia se fundamenta en la finalidad esencial del artículo científico, que es el comunicar los resultados de investigaciones, ideas y debates en una forma clara, precisa, concisa y fidedigna.

En muchas ocasiones los artículos publicados en las revistas latinoamericanas presentan deficiencias en esos aspectos. Por ello, el objetivo de este trabajo es llamar la atención sobre las deficiencias encontradas en la metodología experimental descrita y aplicada en algunas de las revistas científicas, editadas entre los años 2000 y 2003 en países de América Latina y el Caribe.

\section{MATERIALES Y MÉTODOS}

Se seleccionaron aleatoriamente 25 revistas científicas agropecuarias del área latinoamericana, del fondo de información científico técnica de la biblioteca del Instituto de Ciencia Animal, publicadas en el periodo del 2000 al 2004, y se revisaron todos los artículos publicados en ellas.

En ellas se revisaron los materiales y métodos y los resultados de todos los artículos, para cuantificar las deficiencias existentes en la metodología experimental utilizada y descrita, siguiendo los criterios [2], [8] y [7], así como los resultados mal presentados y discutidos, en [9].

Se calcularon los porcentajes correspondientes a los errores más frecuentes y se presentan tablas con aquellos aspectos que tuvieron valores iguales o superiores al $10 \%$.

\section{RESULTADOS Y DISCUSIÓN}

En cualquier proceso de investigación resulta imprescindible utilizar una metodología experimental adecuada y resulta obligatorio describir el diseño de la investigación y explicar cómo se llevó a la práctica, y justificar la elección de métodos y técnicas de forma tal que el lector competente pueda repetir el estudio. Se debe presentar la descripción según la secuencia que siguió la investigación: diseño, población y muestra, variables, toma de dato y análisis. También se deben presentar los resultados del estudio mencionando los resultados o hallazgos relevantes (incluso los contrarios a la hipótesis), incluyendo detalles suficientes para justificar las conclusiones. En la discusión, por último, se deben mostrar las relaciones entre los hechos observados y establecer conclusiones al inferir o deducir una verdad y responder a la pregunta de la investigación planteada en la introducción.

Los porcentajes de errores más frecuentes en el acápite de materiales y métodos se presentan en la Tabla 1. El mayor valor $(62 \%)$ correspondió a la no especificación del paquete estadístico usado para el procesamiento de la información. Parece ser que éste no es un requisito obligatorio en algunas de las revistas revisadas. No obstante, se considera como un elemento importante, pues los sistemas estadísticos de más prestigio son conocidos por los especialistas y garantizan el correcto procesamiento de la información.

Iguales porcentajes (30\%) correspondieron a la descripción incorrecta del diseño experimental utilizado y a la no especificación de la transformación de la variable usada ni señalar la prueba de comparación múltiple de medias.

Una incorrecta descripción del diseño experimental puede ser causada por la ausencia de asesoría estadística en el desarrollo de la investigación o por la aplicación incorrecta del diseño experimental usado. En ambos casos los resultados obtenidos pueden y deben ser cuestionados, más aún cuando cualquier investigador o técnico tiene posibilidades de procesar información en una computadora. Cuando el diseño experimental no es correcto, pueden quedar sin control algunos factores que aumentarán el valor del error experimental y, como consecuencia, no se encontraron aquellas diferencias significativas, o importantes, entre los tratamientos que se prueban. 
Las deficiencias relacionadas con la transformación de las variables y la aplicación de las pruebas de comparación múltiple de medias, mostraron un alto porcentaje $(30 \%)$. La transformación de una variable medida es necesaria cuando no se cumplen algunas de las hipótesis de base de los modelos para los diseños experimentales, por lo que, si este aspecto no es correctamente tratado, ninguna de las bondades de este método estadístico es válida. Son cinco las pruebas de comparación múltiple de medias más usadas, y cada una posee diferentes regiones de rechazo de la hipótesis. Ello determina que las medias de tratamientos que resultan significativas no sean las mismas, y que es necesario conocer con qué prueba se ha trabajado.

Las deficiencias correspondientes a limitar la descripción de la metodología estadística a la descripción al método o métodos de muestreo usado, así como a la descripción incorrecta de los métodos estadísticos complejos (modelado matemático y técnicas multivariadas entre otros), aunque presentan porcentajes más bajos no dejan de tener importancia. En el primer caso no se cumple con el requisito de presentar toda la metodología usada y el segundo es muy peligroso, por cuanto también puede llevar a conclusiones falsas.

En la Tabla 2 se presentan los sucesos de importancia en los resultados y discusión. El mayor porcentaje de deficiencias estuvo relacionado con la información de las tablas, cuando sólo se presentan las medias de los tratamientos probados y no se acompañan de sus correspondientes errores estándar u otro indicador de la variabilidad (51\%). Este problema, que se presenta con mayor frecuencia en los últimos tiempos, es grave, por cuanto la no presentación de estos estadígrafos no permite tener una idea correcta de la precisión y exactitud de los valores estimados. También se observó un alto porcentaje (33\%) de artículos donde las tablas no tenían toda la información necesaria, entre ellas, la ausencia de diferencias significativas encontradas, la no especificación de la prueba de comparación múltiple de medias aplicada, presentación de los indicadores sin la correspondiente unidad de medida, identificación de los tratamientos con números y siglas, entre otras.

Un error también frecuente es presentar y discutir los resultados sin tomar en consideración los resultados estadísticos obtenidos. Para las explicaciones, se utilizan frases como "el tratamiento $\mathrm{x}$ aumentó con relación a ...", “... la tendencia es a disminuir...", lo que puede llevar a manifestar cierta contradicción o incertidumbre entre lo que aparece en la tabla y el texto.

También se hace incorrecto uso de la prueba T-Student, que ha sido definida para realizar la comparación de dos medias a priori. Sin embargo, su utilización cuando son más de dos medias a comparar no es adecuada. En estos casos se deben usar otras pruebas de comparación múltiple.

Tabla 1. Porcentajes de deficiencias en los materiales y métodos.

\begin{tabular}{|l|c|}
\hline Descripción & Valor \% \\
\hline No se especifica el paquete estadístico usado para el procesamiento de la información & 62 \\
\hline Descripción incorrecta del diseño experimental & 30 \\
\hline No aparecen especificadas la transformación, ni prueba de comparación múltiple de medias & 30 \\
\hline No refieren análisis estadístico & 21 \\
\hline Sólo hace referencia al método de muestreo usado & 11 \\
\hline Inapropiada descripción de métodos estadísticos complejos & 11 \\
\hline
\end{tabular}


Tabla 2. Porcentajes de deficiencias en los resultados y discusión.

\begin{tabular}{|l|c|}
\hline Descripción & Valor \% \\
\hline $\begin{array}{l}\text { En las tablas se informa sólo las medias de los tratamientos, } \\
\text { sin reportar el correspondiente error estándar }\end{array}$ & 51 \\
\hline Las tablas no contienen los elementos estadísticos necesarios & 33 \\
\hline Se presentan los resultados sin tomar en consideración los análisis estadísticos realizados & 30 \\
\hline Comparaciones de más de dos medias a través de la prueba T-Student & 22 \\
\hline Resultados incompletos de la aplicación del análisis de regresión & 20 \\
\hline Interpretación incorrecta de la aplicación de métodos estadísticos complejos. & 10 \\
\hline
\end{tabular}

El porcentaje de artículos en que los resultados del análisis de regresión se presentaron en forma incompleta fue alto $(20 \%)$. Estas dificultades se relacionaron con la interpretación errónea del coeficiente de correlación (r) y coeficiente de determinación $\mathrm{R}^{2}$ [3]. En el caso de presentar el modelo ajustado no aparecen los errores estándar de los coeficientes estimados para el modelo, ni el nivel de significación de la hipótesis probada. Para la selección del modelo de mejor ajuste es necesario tomar en consideración la magnitud del cuadrado medio del error de la regresión cuando el modelo es alineal o complejo.

Por último la presentación de trabajos con aplicaciones de métodos estadísticos complejos va en aumento, lo que es lógico y correcto. Sin embargo, estos resultados deben ser bien discutidos y presentados correctamente, lo que no es así en un elevado número de trabajos $(10 \%)$.

En [5], al hacer un estudio sobre los errores frecuentes de los artículos enviados a la "Revista Cubana de Ciencias Agrícolas" señalaron, entre otros: títulos que no reflejan el contenido del artículo o muy extensos, carencia de información en el resumen, introducción larga, materiales y métodos que no permiten repetir la investigación, deficiente discusión de los resultados, y problemas con las referencias.

Estos autores manifestaron que uno de los problemas de mayor relevancia estaba relacionado con el tratamiento estadístico de la información, lo que repercutía en que las conclusiones no correspondieron íntegramente con los resultados presentados.

Esta situación fue mucho más favorable en el período 1998-2003, debido a que el arbitraje de los artículos se utilizó como una vía de superación y formación de los autores. De esta manera se logró disminuir las deficiencias en los trabajos enviados a la referida revista [4].

Los resultados de este estudio y que corresponden al análisis de 25 revistas de América Latina y el Caribe guardan estrecha relación con la información obtenida a partir de los artículos enviados a la Revista Cubana de Ciencias Agrícolas. Esto nos impone la necesidad de buscar y emplear métodos que permitan incrementar la calidad e impacto de los artículos publicados, mediante un adecuado y correcto procesamiento estadístico de la información obtenida.

\section{CONCLUSIONES}

El análisis realizado nos permite concluir que para lograr parámetros de calidad en las publicaciones científicas se debe:

- Incrementar el nivel de conocimiento de los investigadores y docentes en las metodologías estadísticas.

- Incorporar en los consejos editoriales especialistas en estadística que detecten y 
ayuden a resolver las deficiencias en este sentido.

- Incluir en las normas editoriales instrucciones relacionadas con los aspectos estadísticos.

- Promover grupos y foros de discusión sobre estos temas.

- Utilizar las Tecnologías de Información y Comunicación (TIC) para integrar estos esfuerzos y poder alcanzar la calidad de las revistas agropecuarias en la región.

\section{REFERENCIAS}

[1] G.O. Alonso. "Hacia el establecimiento del índice latinoamericano de publicaciones científicas Latindex". Biblioteca Universitaria, Boletín Informativo de la Dirección General de Bibliotecas. Nueva Época, 1 (2): 53-58, julio-diciembre, 1998. Disponible en:

http://www.dgbiblio.unam.mx/servicios/dgb/ publicdgb/bole/fulltext/voll2/default.html [consulta: 5 de septiembre de 2004].

[2] W.G. Cochran y G.M. Cox. "Diseños Experimentales” Editorial F. Trillas, S. A. México, 1965.

[3] P. W. Charles. "Why Regression", Biometrics Vol. 2 No. 6, 1946.
[4] R.S. Herrera. "Los errores de los artículos: Una vía de educación y formación de los autores". V Encuentro de Editores de Revistas Científicas, La Habana, 2004, CD-ROM.

[5] R.S. Herrera, V. Torres y M. Ribas. "Errores frecuentes en la escritura del artículo científico". II Encuentro de Editores de Revistas Científicas. La Habana, 1998, CD-ROM.

[6] M.C. Ratto y A.B Dellamea. "Difusión, acceso y visibilidad de publicaciones científicas seriadas de Iberoamérica: el sistema Latindex". Redacción y Comunicación Científicas. Universidad de Buenos Aires. Argentina, 2001.

[7] I. B. M. Sampaio. "Estadística Aplicada á Experimentação Animal. Fundação de Ensino e Pesquisa em Medicina Veterinária e Zootecnia. Belo Horizonte, 1998.

[8] R.G.D. Steel y J. H. Torrie. "Bioestadística: Principios y Procedimientos". McGraw-Hill/ Interamericana de México, S.A. de C.V., 1992.

[9] V. Torres. "Preponderancia de los métodos estadísticos en el índice de impacto (SCI) de las revistas científicas". III Encuentro de Editores de Revistas Científicas. La Habana, 2002, CD-ROM. 